\title{
Dynamics of a physiologically structured population in a time-varying environment
}

Heilmann, Irene Louise Torpe; Starke, Jens; Andersen, Ken Haste; Thygesen, Uffe Høgsbro; Sørensen, Mads Peter

\author{
Published in: \\ Ecological Complexity
}

Link to article, DOI:

10.1016/j.ecocom.2016.10.004

Publication date:

2016

Document Version

Peer reviewed version

Link back to DTU Orbit

Citation (APA):

Heilmann, I. L. T., Starke, J., Andersen, K. H., Thygesen, U. H., \& Sørensen, M. P. (2016). Dynamics of a physiologically structured population in a time-varying environment. Ecological Complexity, 28, 54-61.

https://doi.org/10.1016/j.ecocom.2016.10.004

\section{General rights}

Copyright and moral rights for the publications made accessible in the public portal are retained by the authors and/or other copyright owners and it is a condition of accessing publications that users recognise and abide by the legal requirements associated with these rights.

- Users may download and print one copy of any publication from the public portal for the purpose of private study or research.

- You may not further distribute the material or use it for any profit-making activity or commercial gain

- You may freely distribute the URL identifying the publication in the public portal 


\title{
Dynamics of a physiologically structured population in a time-varying environment
}

\author{
Irene Heilmann ${ }^{\mathrm{a}, \mathrm{b}}$, Jens Starke ${ }^{\mathrm{a}, \mathrm{c}}$, Ken H. Andersen ${ }^{\mathrm{b}}$, Uffe \\ Høgsbro Thygesen ${ }^{\mathrm{b}}$, and Mads Peter Sørensen ${ }^{\mathrm{a}}$ \\ a Department of Applied Mathematics and Computer Science, \\ Technical University of Denmark \\ ${ }^{\mathrm{b}}$ Center for Ocean Life, National Institute of Aquatic Resources, \\ Technical University of Denmark \\ ${ }^{\mathrm{c}}$ School of Mathematical Sciences, Queen Mary University of \\ London
}

\begin{abstract}
Physiologically structured population models have become a valuable tool to model the dynamics of populations. In a stationary environment such models can exhibit equilibrium solutions as well as periodic solutions. However, for many organisms the environment is not stationary, but varies more or less regularly. In order to understand the interaction between an external environmental forcing and the internal dynamics in a population, we examine the response of a physiologically structured population model to a periodic variation in the food resource. We explore the addition of forcing in two cases: A) where the population dynamics is in equilibrium in a stationary environment, and B) where the population dynamics exhibits a periodic solution in a stationary environment. When forcing is applied in case A, the solutions are mainly periodic. In case B the forcing signal interacts with the oscillations of the unforced system, and both periodic and irregular (quasi-periodic or chaotic) solutions occur. In both cases the periodic solutions include one and multiple period cycles, and each cycle can have several reproduction pulses.
\end{abstract}

Keywords: Structured population model · Periodic variation · Bifurcation diagram

\section{Introduction}

Environmental variations are evident drivers of abundance and succession in temperate ecosystems [15]. Variations in the environment are changes in the abiotic environment, such as light and temperature, as well as in the biotic environment, such as presence of prey and predators. These conditions together affect processes such as growth and mortality for the individual. Variations may occur on very different time scales, ranging from diel patterns in light levels, over weather phenomena on weekly time scales and seasonal patterns in 
temperature, to decadal fluctuations such as governed by the North Atlantic Oscillation (NAO). Some of these variations are strictly periodic whereas others are less regular.

Here, we consider a model inspired by planktonic crustaceans. Planktonic crustaceans, such as daphnia in fresh water or copepods in marine environments, are subjected to environmental changes on daily to yearly time scales. They are multicellular organisms with a complex life cycle. The weight of each egg reaches approximately $1 \%$ of the individual biomass, and the eggs hatch to become nauplii which molt through successive stages to become adults. In the adult stage, all acquired food is going to survival (including building reserves) and to reproduction. The extended life implies that environmental variability can have complex effects on the organisms' life history. Planktonic crustaceans are a key link between primary producers and higher trophic levels (fish). An understanding of this link requires an understanding of how their life cycle is affected by a varying environment.

Existing literature concerning environmental variation in planktonic ecosystems has often modelled unstructured populations. Then, the ecosystem is represented as a Nutrient-Phytoplankton-Zooplankton (NPZ) model [8], where the zooplankton, representing planktonic crustaceans, is described by a single state variable representing their abundance or biomass. In [7], for example, is considered a seasonal forcing that acts through influence of sunlight on photosynthesis of the phytoplankton. Studies of forcing in predator-prey models demonstrates a very rich dynamical behaviour with period doublings, quasi-periodic and chaotic dynamics, as well as coexisting attractors [17, 20, 22]. These phenomena often arise in systems that oscillate even in the absence of forcing and for large forcing amplitudes. While these models illustrate the dynamics of forced systems of unicellular organisms well, they are unable to describe the life history of multi-cellular organisms such as planktonic crustaceans.

Physiologically structured population models are a well investigated class of models [6, 16]. They are suited for modelling species when mass-specific rates of biomass productions and maintenance change significantly with the size of individuals [6]. In a constant environment, physiologically structured models can show equilibrium solutions as well as periodic solutions. The periodic solutions arise as a results of predator-prey dynamics or competition between adults and juveniles [6]. In [23], a physiologically structured population model is considered with seasonal variation of environmental conditions as well as pulsed reproduction at a fixed time of year. The emphasis is on mortality and conditions for survival. Pulsed reproduction can cause similar effects to forcing, such as multiple year cycles, without explicit changes in environmental conditions. An example of this is found in [19] for a physiologically structured population.

The aim of this paper is to analyze the response of a physiologically structured population subjected to a periodic variation of the resource production. We regard the periodic variation of the environment as a driving force of the population. If the system of the population model oscillates in a constant environment, the addition of a forcing term implies an interaction between two (or more) frequencies, and the system resembles that of a forced oscillator. Our approach is to take a well-known physiologically structured model for a daphnia population and its algae resource [5]. We look at two basic cases: one where the system oscillates even in the absence of forcing, and another where it reaches 
a stable equilibrium; and investigate the effect of adding forcing to each case. The emphasis is on the different dynamics that can occur.

\section{Model}

We use the Kooijman-Metz model, [4, 11], of a daphnia population feeding on an algae resource. Individual daphnia allocate energy from food intake between growth, basic metabolism and reproduction. The daphnia population is size structured, i.e., the model distinguishes between individuals of different size, whereas the algae resource is unstructured. The model was first presented in [11], where it is compared to experimental data, and in [4] the dynamic properties of the model are explored. We employ the specific formulation of the Kooijman-Metz model given in [5]:

$$
\begin{gathered}
\frac{\partial n(x, t)}{\partial t}+\frac{\partial g(F, x) n(x, t)}{\partial x}=-\mu n(x, t), \\
\frac{\mathrm{d} F(t)}{\mathrm{d} t}=R(F)-\int_{x_{0}}^{x_{m}} I(F, x) n(x, t) \mathrm{d} x, \\
g\left(F, x_{0}\right) n\left(x_{0}, t\right)=\int_{x_{0}}^{x_{m}} b(F, x) n(x, t) \mathrm{d} x .
\end{gathered}
$$

Equation (1a) describes the time evolution of the daphnia population density distribution $n(x, t)$ over the length $x$ of an individual at time $t$. The daphnia have a somatic growth rate $g(F, x)$ and a constant mortality rate $\mu$. The concentration of algae $F(t)$ evolves according to $(1 \mathrm{~b})$; the algae grow at the rate $R(F)$ in the absence of daphnia. The integral in (1b) represents daphnia ingesting algae at the rate $I(F, x)$. The combined reproduction of the daphnia population gives rise to the boundary condition (1c) at $x_{0}$, the length of a daphnia at birth. We will present expressions for the functions in (1) shortly.

The model presented here is specifically adapted to daphnia and algae, but the assumptions and basic mechanisms behind the model could also apply to other species, such as marine copepods. As such, the Kooijman-Metz model represents a broader class of predator-prey models [4]. It resembles the RosenzweigMacArthur model in the sense that without size structure, i.e., if all individuals are assumed to have the same length $x$, the system (1) reduces to the Rosenzweig-MacArthur model, see [4, 5] for details.

In the following, we present the functions entering the system (1). The expressions are based on the daphnia's allocation of energy to different processes, and a detailed derivation is given in [5]. The daphnia's encounter of algae $F$ is proportional to a Holling type II functional response,

$$
h(F)=\frac{F}{F_{h}+F},
$$

where $F_{h}$ is the half-saturation constant. The ingestion rate $I(F, x)$ of daphnia is proportional to the surface area of the individual and to the functional response, giving

$$
I(F, x)=\nu h(F) x^{2},
$$

where $\nu$ is a proportionality constant. The somatic growth rate of daphnia is

$$
g(F, x)=\gamma\left(x_{m} h(F)-x\right),
$$


where $x_{m}$ is an upper bound on the length a daphnia can achieve, and $\gamma$ is a proportionality constant. The maximum length of an individual thus depends on the available energy. Individuals become mature and start to reproduce when they reach a specific length $x_{j}$. Individuals above this length allocate a fixed proportion of their energy intake to reproduction. This gives a birth rate of the form

$$
b(F, x)=\left\{\begin{array}{ll}
r_{m} h(F) x^{2} & \text { for } x \geq x_{j} \\
0 & \text { for } x<x_{j}
\end{array},\right.
$$

where $r_{m}$ is a proportionality constant. Finally, the algae are assumed to have logistic growth $R(F)$ in the absence of predators:

$$
R(F)=\alpha F\left(1-\frac{F}{K}\right) .
$$

Here, $\alpha$ is the specific growth rate of algae and $K$ is the carrying capacity of algae. In the basic model, $\alpha=\alpha_{0}$ is constant. The default parameter values are listed in table 1 and are from [5].

\begin{tabular}{|c|c|c|c|}
\hline Symbol & Value & Unit & Description \\
\hline$x$ & - & $\mathrm{mm}$ & Length of individual \\
\hline$t$ & - & day & Time \\
\hline$F(t)$ & - & $\mathrm{mgC} / \mathrm{L}$ & Density of algae food resource \\
\hline$n(x, t)$ & - & $1 / \mathrm{L}$ & $\begin{array}{l}\text { Number density distribution of } \\
\text { daphnia population }\end{array}$ \\
\hline$x_{0}$ & 0.6 & $\mathrm{~mm}$ & Length at birth \\
\hline$x_{j}$ & 1.4 & $\mathrm{~mm}$ & Length at maturity \\
\hline$x_{m}$ & 3.5 & $\mathrm{~mm}$ & $\begin{array}{l}\text { Largest possible length for any } \\
\text { conditions }\end{array}$ \\
\hline$F_{h}$ & 0.164 & $\mathrm{mgC} / \mathrm{L}$ & Half-saturation resource level \\
\hline$\gamma$ & 0.11 & $1 /$ day & Somatic growth constant \\
\hline$r_{m}$ & 1.0 & $1 /\left(\right.$ day $\left.\cdot \mathrm{mm}^{2}\right)$ & Birth rate constant \\
\hline$\nu$ & 0.007 & $\mathrm{mgC} /\left(\right.$ day $\left.\cdot \mathrm{mm}^{3}\right)$ & Ingestion constant \\
\hline$\alpha_{0}$ & 0.5 & $1 /$ day & Specific resource growth rate \\
\hline$K$ & {$[0 ; 1]$} & $\mathrm{mgC} / \mathrm{L}$ & Carrying capacity of resource \\
\hline$\mu$ & {$[0 ; 0.3]$} & $1 /$ day & Mortality \\
\hline$a_{f}$ & {$[0 ; 1]$} & - & Strength of forcing \\
\hline$T_{f}$ & {$[5 ; 100]$} & day & Period of forcing \\
\hline
\end{tabular}

Table 1: Variables and parameter values.

The system (1) can exhibit four types of solutions [4,5] depending on daphnia mortality $\mu$ and carrying capacity $K$ of the algae. The daphnia population can go extinct, i.e., $n(x, t)=0$ for all $x$ and $t$, or reach a positive equilibrium, i.e., $n(x, t)=\tilde{n}(x)$ for all $t$, with a positive number of individuals in the daphnia population. Furthermore, the system can show periodic solutions, categorized as either cohort cycles or predator-prey cycles [5]. For cohort cycles, the population is dominated by a cohort of individuals concentrated around a single length $x$. When the individuals in the cohort grows larger and reaches maturity, they give birth to a new cohort that eventually takes over. The mechanism in predatorprey cycles is that first the amount of prey increases, which leads to an increase 
of the predator. This in turn causes the prey to diminish, which leads to a decrease of the predator, and then the cycle repeats itself. In our case, the daphnia population plays the role of the predator and the algae act as the prey. Predator-prey cycles are also called paradox of enrichment oscillations [18], and are similar to the oscillations in Lotka-Volterra models. The transition between cohort and predator-prey cycles in the model (1) is gradual and some solutions have characteristics of both types. The existence and stability of the different solution types depends on the parameter values.

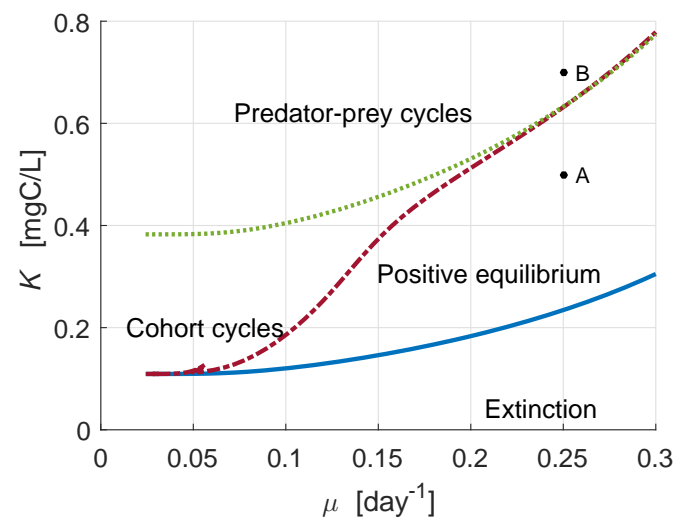

Figure 1: Bifurcation diagram for the system (1) in $(\mu, K)$-parameter space. The solid blue line defines the boundary of existence of the positive equilibrium solution, and the dash-dot red line shows when this solution becomes unstable. Below the solid blue line only the equilibrium solution with zero daphnia exists, and above the dash-dot red line periodic solutions emerge. The dotted green line indicates an approximate boundary between the cohort and predator-prey cycles. The points marked A and B represents the two parameter cases we will use.

Figure 1 is a bifurcation diagram showing regions where different solution types exist and are stable. The parameters varied are the daphnia mortality $\mu$ and the carrying capacity $K$ of the algae. We have used the criteria presented in $[4,5]$ to distinguish between solution types. The solutions to the equations are continued using the continuation software CoCo [2, 3] for MATLAB [14]. The solid blue line in figure 1 shows the existence boundary for the equilibrium solution with a non-zero number of daphnia. Below this line, the only solution is the equilibrium with zero daphnia. Based on the linear stability analysis in $[4,5]$, the dash-dot red line depicts where the positive equilibrium solution looses stability and a periodic solution emerges, corresponding to a Hopf bifurcation. An approximate separation between cohort cycles and predator-prey cycles is indicated by the dotted green line. The transition is not characterized by a bifurcation, but by a descriptive criterion derived in [4]. The criterion comes from comparing the stability conditions for the structured model to those for a corresponding unstructured model. The Hopf line has two small hairpin turns close to $\mu=0.05$, which differs from a corresponding bifurcation diagram in [5]; presumably this is because we use a continuation approach that captures more details. We will use the parameter values indicated by the points $\mathrm{A}\left(\mu=0.25\right.$ day $^{-1}$, 
$K=0.5 \mathrm{mgC} / \mathrm{L})$ and $\mathrm{B}\left(\mu=0.25 \mathrm{day}^{-1}, K=0.7 \mathrm{mgC} / \mathrm{L}\right)$ as representative cases for an equilibrium solution and a periodic solution, respectively.

The discontinuity of the birth rate function $b(F, x)$ in equation (4) at $x=x_{j}$, leads to ambiguity of certain solutions with cohort cycles, but otherwise the model is well-posed [21]. We will not investigate solutions with cohort cycles, so we expect the solutions are uniquely defined, even when the forcing term introduces a smooth variation of parameters.

\section{$2.1 \quad$ Forcing}

We proceed to introduce variation of the environment in the model (1). This can be done by letting one or more of the parameters vary with time. In [17], they investigate six different choices of parameters for applying periodic variation to the Rosenzweig-MacArthur model, and conclude that all six possibilities give qualitatively similar results. Here, we follow the approach by $[9,20,22]$ and introduce the forcing via the resource growth rate, $\alpha$, to reflect variations in for example light level and nutrient conditions. Numerical investigations reveal that this parameter has very little influence on the bifurcation diagram in figure 1 close to the points $\mathrm{A}$ and $\mathrm{B}$, which is consistent with the analysis in [4]. Therefore, the system does not switch between regions of the bifurcation diagram when $\alpha$ changes with time, and the effects of forcing will not be mixed with qualitative changes in the basic model. For a system with no daphnia, forcing of the algae growth rate, $\alpha$, results in the algae stabilizing at the carrying capacity $K$. However, the systems we are considering are never close to extinction of daphnia, so we do not expect to see such equilibrium solutions.

The environmental variation is implemented as a sinusoidal change of the algae growth rate, $\alpha$, that now becomes time-dependent:

$$
\alpha(t)=\alpha_{0}\left(1+a_{f} \sin \left(\frac{2 \pi}{T_{f}} t\right)\right)
$$

We keep the mean value fixed to the default value, $\alpha_{0}$, from the original model. The amplitude, $a_{f} \in[0,1]$, is a measure of the magnitude of the variations, and $T_{f}$ defines the period of the variations.

We are interested in the relation between the time scale of the forcing, $T_{f}$, and a time scale of the internal dynamics of the unforced system, $T_{s y s}$, so we introduce the ratio: $\theta=T_{f} / T_{\text {sys }}$. If the unforced system (1) exhibits stable oscillations, we define the time scale $T_{s y s}$ as the period of these oscillations, and compute it directly from the solution. The parameter values in case $\mathrm{B}$ give an oscillatory solution with an estimated period of $T_{\text {sys }} \approx 27.6$ days. When the unforced population dynamics display damped oscillations towards a stable equilibrium, we define the time scale $T_{s y s}$ as the quasi-period of such smallamplitude transient oscillations. To estimate this value, we numerically compute the Jacobian of the discretized system at the equilibrium; the discretization of the system is described in section 2.2. The quasi-period is determined from the imaginary part of the dominating eigenvalue of the Jacobian. An example of such dynamics arises for the parameter values in case A where the period is approximately $T_{\text {sys }} \approx 27.4$ days. We have varied the forcing period in the range $T_{f} \in[5,100]$ days as simulations revealed the most interesting dynamics for these values. 


\subsection{Numerical integration}

For the Kooijman-Metz model in (1), analytic solutions exist for the equilibrium solutions, but not for the periodic solutions. With the addition of forcing, the system becomes more complex and hence we concentrate on numerical solutions. We use the method of lines [10, 12], and discretize the partial differential equation (PDE) given by (1a) in the size variable $x$. This leaves a system of ordinary differential equations (ODEs) in time $t$, which we integrate with a fourth order Runge-Kutta method using MATLAB's ODE solver ode45 [14]. As equation (1a) is a type of advection or transport equation with velocity $g(F, x)$, we discretize with a first order upwind scheme $[10,12]$, where the upwind direction changes with the sign of $g(F, x)$. Simulations indicates that 300 linearly distributed discretization points is ample for resolving the solution, and we use this number in all computations. For each set of parameter values, the system is integrated in time until the solution has settled to a stable stationary or periodic state.

\section{Results}

\subsection{Population without self-oscillations}

In this section we examine the effect of the forcing (6) to the system (1) with the parameters in case $\mathrm{A}\left(\mu=0.25\right.$ day $^{-1}, K=0.5 \mathrm{mgC} / \mathrm{L}$; see figure 1$)$. Without forcing, this system has a stable equilibrium solution with a non-zero daphnia population, see figure $2 \mathrm{a}$, and transient oscillations with a period of $T_{\text {sys }} \approx 27.4$ days, see section 2.1. A typical solution to the forced system is stable periodic oscillations, that resembles predator-prey cycles, see figure $2 \mathrm{~b}$.

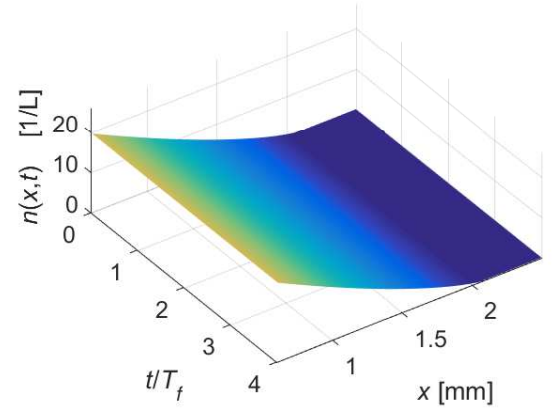

(a) $a_{f}=0$

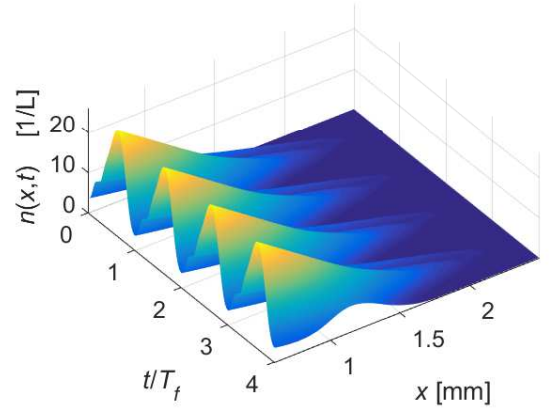

(b) $a_{f}=0.4$

Figure 2: Examples of solutions for the population density distribution $n(x, t)$ in case $\mathrm{A}\left(\mu=0.25 \mathrm{day}^{-1}, K=0.5 \mathrm{mgC} / \mathrm{L}\right)$. (a) The unforced system has a stable stationary solution. (b) The forced system, with forcing amplitude $a_{f}=0.4$ and forcing period $T_{f}=30$ days, has a stable periodic solution with period $T_{\text {sol }}=T_{f}$.

First, the forcing period is kept fixed at $T_{f}=30$ days, which is close to the time scale of the unforced system $T_{\text {sys }}$, giving the time scale ratio $\theta \approx 1.1$. For this period, we consider a series of examples with increasing value of the forcing amplitude $a_{f}$, see figure 3 . For each amplitude, the system is integrated until the 
solution has settled to a stable stationary or periodic state. To characterize the population distribution $n(x, t)$ with a single measure (for each point in time), we use the birth rate of the entire population,

$$
B(t):=\int_{x_{0}}^{x_{m}} b(F, x) n(x, t) \mathrm{d} x .
$$

This is also the right hand side of the boundary condition (1c). Initially, the solution locks in phase with the forcing, and the amplitude of the birth rate increases with the forcing amplitude $a_{f}$ (figure 3a). Increasing the forcing amplitude further leads to a period doubling (figure $3 \mathrm{~b}$ ). After the period doubling, the solution changes gradually from having one dominating birth pulse for every forcing period $T_{f}$, to having one for every two forcing periods. This is a typical pattern for case A when keeping the forcing period $T_{f}$ fixed and varying the forcing amplitude $a_{f}$.

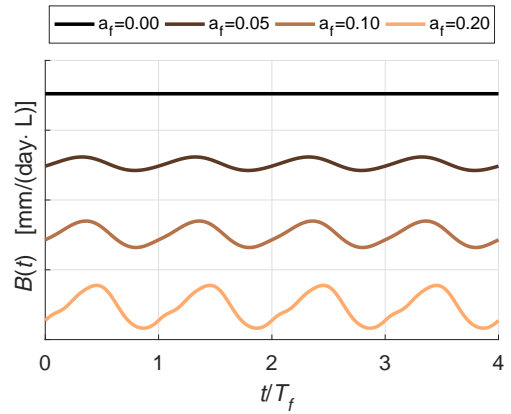

(a)

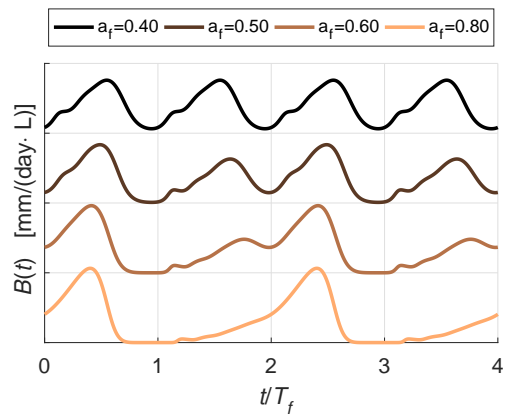

(b)

Figure 3: Combined birth rate $B(t)$ of the population in case A $\left(\mu=0.25\right.$ day $^{-1}$, $K=0.5 \mathrm{mgC} / \mathrm{L}$ ). The forcing period is kept constant for a time scale ratio of $\theta \approx 1.1$, while the forcing amplitude $a_{f}$ is varied. Each section on the vertical axis represents the interval $[0 ; 6] \mathrm{mm} /($ day $\cdot \mathrm{L}$ ). (a) The non-oscillating solution locks to the phase of the forcing and the amplitude of the birth rate increases with $a_{f}$. (b) As $a_{f}$ increases further, the birth rate changes in a period doubling.

For a more detailed exploration of the population's response to changes in the forcing amplitude, we do a systematic parameter sweep. This entails we repeatedly change the parameter $a_{f}$ a small amount and integrate the system in time until it has settled to a stable stationary or periodic state. The final solution is used as the starting point in the next iteration. Experimenting showed that the transient behavior has vanished after 100 forcing periods or at least 3000 days. To have a sample of the steady state solution, the solution is integrated for 12 forcing periods or at least 360 days after the transient has vanished. For oscillatory solutions with a period up to four forcing periods, this gives a sample of at least three whole periods. First, a forward sweep runs through the values $a_{f}=0,0.1, \ldots, 1$ and then a backward sweep goes through the same values, but in opposite direction. To get an overview of the results, we characterize each solution with a few measures, which are described in the following. Figure 4 shows the results of a parameter sweep where the forcing period is again kept constant at $T_{f}=30$ days, giving the time scale ratio $\theta \approx 1.1$. 


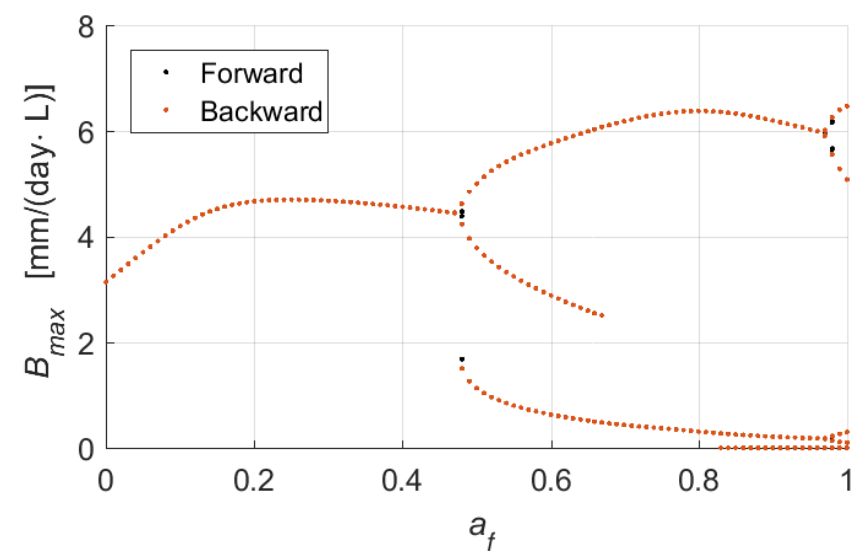

(a)

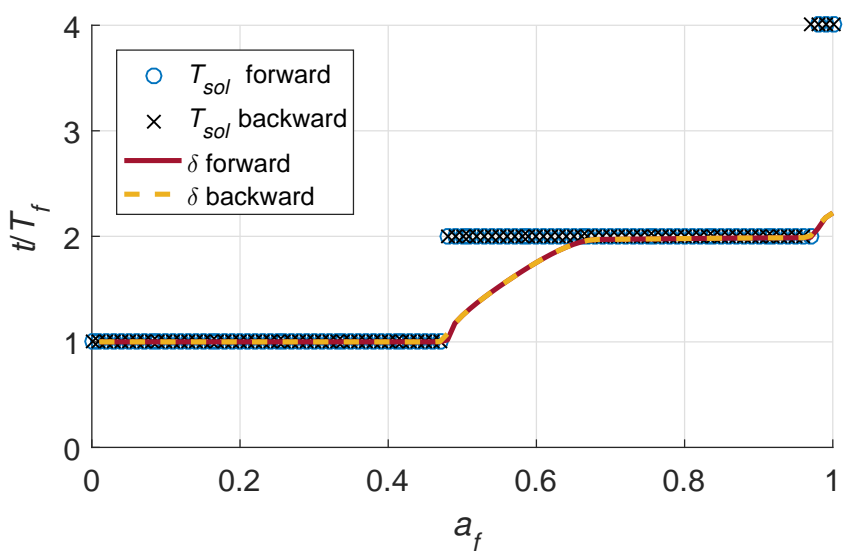

(b)

Figure 4: Parameter sweep in the forcing amplitude $a_{f}$ for case $\mathrm{A}(\mu=$ $\left.0.25 \mathrm{day}^{-1}, K=0.5 \mathrm{mgC} / \mathrm{L}\right)$ with a forcing period corresponding to the time scale ratio $\theta \approx 1.1$. The figures indicate a period doubling at $a_{f} \approx 0.47$ and $a_{f} \approx 0.97$. (a) The local maxima $B_{\max }$ of the combined birth rate for each value of $a_{f}$. (b) The period of the solution, $T_{\text {sol }}$, and the average time between birth pulses, $\delta$.

The first characteristic measure of the solutions is the local maxima $B_{\max }$ of the birth rate $B(t)$. For each iteration in the parameter sweep the values of all the local maxima, occurring after the transient behavior, are recorded and plotted against the forcing amplitude $a_{f}$, see figure $4 \mathrm{a}$. In other words, for each peak in the birth rate $B(t)$ there is a dot indicating the height of the peak. This gives multiple points for each value of $a_{f}$, where some or all the points may coincide. The birth rate has a single repeated pulse for $a_{f}<0.47$, indicating a periodic solution. However, the period of the cycle cannot be determined from this figure, as the time steps between the maxima are not shown. At $a_{f} \approx 0.47$, the single branch divides into two, indicating a period doubling. Along with 
the period doubling, a third branch of maxima appears clearly below the other branches. Comparing with figure 3, this branch is identified as small extra peaks on the function $B(t)$.

Another characteristic measure of a solution is its period $T_{\text {sol }}$. The period is determined by sampling the settled solution at time intervals of length $T_{f}$, and comparing the solution at these sample points. This is used to determine whether the solution period is an integer multiple of the forcing period. The solution period $T_{\text {sol }}$ is plotted against the forcing amplitude $a_{f}$, see figure $4 \mathrm{~b}$. The solution period jumps directly from $T_{\text {sol }}=T_{f}$ to $T_{\text {sol }}=2 T_{f}$ confirming there is a period doubling at $a_{f} \approx 0.47$.

We will use one more characteristic measure of a solution. The solution shifts gradually from having one birth pulse every $T_{f}$ to having one pulse every $2 T_{f}$ (figure $3 \mathrm{~b}$ ), and this is not reflected in the solution period that changes abruptly. Therefore, we introduce the average time distance between consecutive birth pulses, denoted $\delta$. To compute the number of birth pulses, the notion of total variation $V_{[a, b]}(B)$, of the birth rate function $B$, is employed,

$$
V_{[a, b]}(B)=\int_{a}^{b}\left|B^{\prime}(t)\right| \mathrm{d} t .
$$

This reflects the sum of all the increases and decreases in the function value $B(t)$ in the interval $[a, b]$. Furthermore, the amplitude $A_{[a, b]}(B)$ of the birth rate function $B(t)$ taken for the interval $[a, b]$ is given by

$$
2 A_{[a, b]}(B)=\max _{t \in[a, b]} B(t)-\min _{t \in[a, b]} B(t) .
$$

We define the number of (full size) birth pulses $P_{[a, b]}(B)$ in a time interval $[a, b]$ as the total variation in units of the amplitude:

$$
P_{[a, b]}(B)=\frac{V_{[a, b]}(B)}{4 A_{[a, b]}(B)} .
$$

The factor 4 comes from considering a continuous function $B(t)$ that is periodic on the interval $[a, b]$. For such a function, the total variation will measure the height of every peak twice, since each peak goes both up and down. In addition, the amplitude will measure half the height of the biggest peak. Dividing with the length of the time interval gives the average number of birth pulses per time, $\Gamma=P_{[a, b]}(B) /(b-a)$. The inverse value, $\delta=\Gamma^{-1}$, corresponds to the average time between two full birth pulses,

$$
\delta=\frac{b-a}{P_{[a, b]}(B)} .
$$

The length of the time interval, $\Delta t=b-a$, should be an integer multiple of the forcing period $T_{f}$, and for periodic solutions it should also be an integer multiple of the solution period $T_{\text {sol }}$. Further, the solution is assumed to have reached a stable stationary or periodic state at time $t=a$. The value of $\delta$ is computed for each iteration in the parameter sweep, see figure $4 \mathrm{~b}$. After the period doubling at $a_{f} \approx 0.47$, the average time between birth pulses changes gradually from $\delta=T_{f}$ to $\delta=2 T_{f}$.

Combining the information from the different measures of the solution, we summarize the results of the parameter sweep for the time scale ratio $\theta \approx 1.1$ 
(figure 4). The solution locks in phase with the forcing for $a_{f}<0.47$ with a single pulse in each forcing period, $\delta=T_{f}$. At $a_{f} \approx 0.47$, there is a period doubling followed by a gradual change in the solution to a birth pulse every $\delta=2 T_{f}$. At $a_{f} \approx 0.97$, there is another period doubling resulting in a period of $4 T_{f}$.

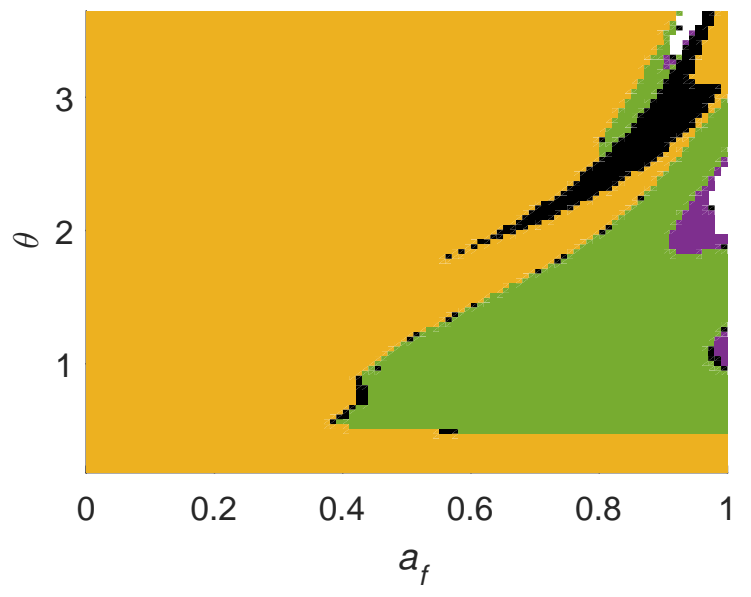

Figure 5: Bifurcation diagram for case $\mathrm{A}\left(\mu=0.25\right.$ day $\left.^{-1}, K=0.5 \mathrm{mgC} / \mathrm{L}\right)$ with parameters for the forcing amplitude $a_{f}$ and the time ratio $\theta=T_{f} / T_{\text {sys }}$. The colors indicate the solution period $T_{\text {sol }}$ in units of $T_{f}, T_{s o l}=T_{f}$ is orange, $T_{\text {sol }}=2 T_{f}$ is green, and $T_{\text {sol }}=4 T_{f}$ is purple. White indicates $T_{\text {sol }}>4 T_{f}$ or that no period was found, while black indicates the forward solution was different from the backward solution.

Repeating the procedure of a parameter sweep for different forcing periods $T_{f}=5,6, \ldots, 100$ days and combining the results, leads to a bifurcation diagram in the parameters $a_{f}$ and $\theta=T_{f} / T_{\text {sys }}$, see figure 5 . For all forcing periods the solution immediately phase locks to the forcing when the forcing amplitude is increased above 0 . There are no period doublings for amplitudes less than $a_{f} \approx 0.37$ or for time scale ratios less than $\theta \approx 0.37$. When $\theta$ increases, period doublings occur for larger values of forcing amplitude $a_{f}$.

Transients times become very large close to the period doublings, and hence the forward and the backward solutions do not completely agree, since the simulations are for finite time. Therefore, we observe a hysteresis-like phenomenon, and in the theory of phase transitions this is referred to as critically slowing down [13]. There is also an area with hysteresis (black) for large $a_{f}$ and large $\theta$, where the forward and backward solution are clearly distinct.

\subsection{Self-oscillating population}

In this section, we turn our attention to case $\mathrm{B}\left(\mu=0.25 \mathrm{day}^{-1}, K=0.5 \mathrm{mgC} / \mathrm{L}\right.$; see figure 1) and investigate the effect of adding forcing to the system (1), using the same approach as in case A. Without forcing, case B gives an oscillatory solution with predator-prey driven cycles, see figure $6 \mathrm{a}$, and has a period of $T_{\text {sys }} \approx 27.6$ days (see section 2.1 ). In figure $6 \mathrm{~b}$, an example is shown of the forced 
system where the solution locks to the forcing, that is $T_{\text {sol }}=T_{f}$. The solution still resembles the predator-prey cycles from the unforced system, except every other bloom of the population has a larger amplitude.

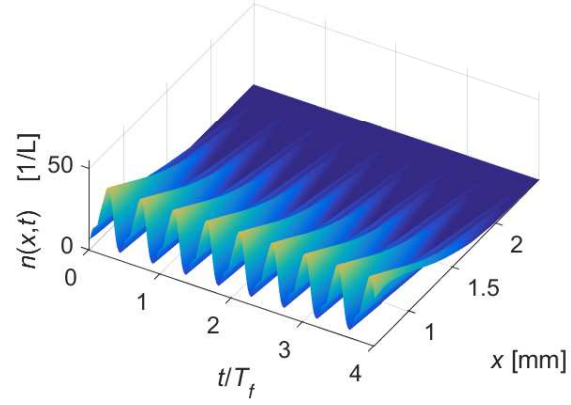

(a) $a_{f}=0$

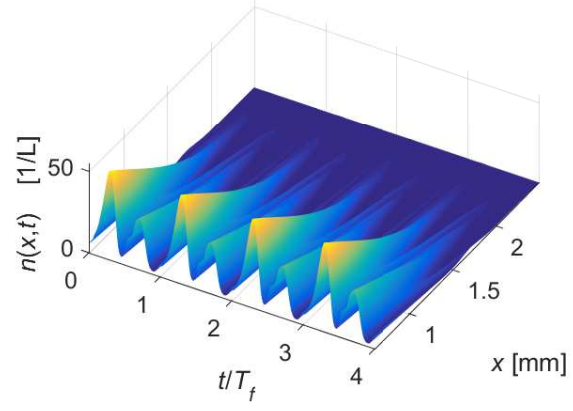

(b) $a_{f}=0.4$

Figure 6: Examples of solutions for the population density distribution $n(x, t)$ in case $\mathrm{B}\left(\mu=0.25 \mathrm{day}^{-1}, K=0.7 \mathrm{mgC} / \mathrm{L}\right)$. (a) The unforced system has a stable periodic solution with period $T_{\text {sol }} \approx T_{f} / 2.2$. (b) The forced system, with forcing amplitude $a_{f}=0.4$ and forcing period $T_{f}=60$ days, has a stable periodic solution with period $T_{\text {sol }}=T_{f}$.

First we consider a forcing with constant period $T_{f}=60$ days, giving the time scale ratio $\theta \approx 2.2$. The combined population birth rate $B(t)$ is plotted for different forcing amplitudes $a_{f}$, see figure 7 . For small forcing amplitude $a_{f}$, the solution resembles the unforced solution, but the pulses are irregular. Increasing $a_{f}$ results in phase locking with the forcing, where the birth rate has two pulses repeating every period. When $a_{f}$ is increased further, the solution first becomes irregular and eventually periodic again, but now there is only one birth pulse in each period.

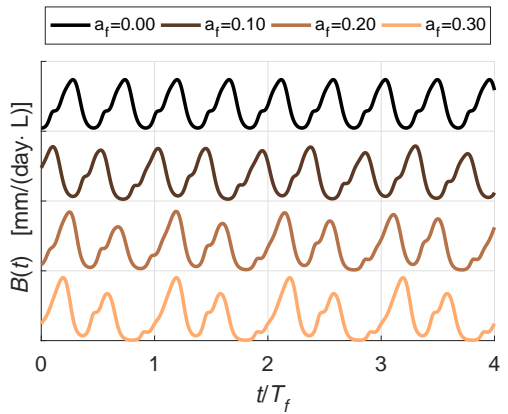

(a)

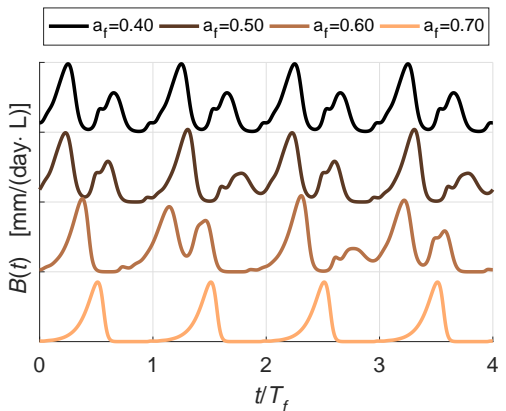

(b)

Figure 7: Combined birth rate $B(t)$ of the population in case $\mathrm{B}\left(\mu=0.25\right.$ day $^{-1}$, $K=0.7 \mathrm{mgC} / \mathrm{L})$. The forcing period is kept constant for a time scale ratio of $\theta \approx 2.2$, while the forcing amplitude $a_{f}$ is varied. Each section on the vertical axis represents the interval $[0 ; 10] \mathrm{mm} /($ day $\cdot \mathrm{L})$. The function changes between being irregular and periodic. 
We proceed as for case A and make a parameter sweep where the forcing amplitude $a_{f}$ is varied in small steps while the forcing period $T_{f}$ is kept fixed. Again the local maxima $B_{\max }$ of the birth rate function $B(t)$ are recorded, as well as the solution period $T_{\text {sol }}$ and the average time $\delta$ between consecutive pulses (figure 8). As the forcing amplitude is increased from zero, $B_{\max }$ shows increasingly irregular behavior and $\delta$ shows increasing distance between the birth pulses. The solution locks to the forcing frequency at $a_{f} \approx 0.30$, and is followed by at least one period doubling. When the amplitude $a_{f}$ is increased further, the periodic solutions break down and the solutions become irregular again. At $a_{f} \approx 0.70$, the irregular solutions are succeeded by another window of periodic solutions containing at least one period doubling. Both intervals of phase locking start with a period of $T_{s o l}=T_{f}$, but the time between birth pulses is less than the forcing period, i.e., $\delta<T_{f}$, in the first interval, whereas it is exactly $\delta=T_{f}$ in the second interval. In the second interval, the forcing frequency completely dominates the solution and the influence of the oscillations in the unforced system are no longer visible. This example displays some features that are also characteristic for other values of $\theta$. These general features include that as the forcing amplitude $a_{f}$ increases, the solution gradually changes from being dominated by the period $T_{s y s}$ of the unforced system to being dominated by the forcing period $T_{f}$.

To construct a bifurcation diagram in the parameters $a_{f}$ and $\theta$, we repeated the simulations for a range of forcing periods $T_{f}=5,6, \ldots, 100$ days, and combined the results, see figure 9 . In large regions of the parameter space, the solutions are irregular or have a period $T_{\text {sol }}>4$ (white area), suggesting the solutions become quasi-periodic or even chaotic. The regions with irregular solutions are interrupted by windows of periodic solutions that spreads out from points on the $\theta$-axis and undergo period doublings when $a_{f}$ increases. Similar to case A, there are hysteresis-like phenomena (black) near the period doublings, because the transient times become very large here. However, there are also larger areas of real hysteresis with existence of two distinct stable states.

Wedges with period $T_{\text {sol }}=T_{f}$ (yellow) are clearly visible going out from $\theta=1,2,3$ and around $\theta=1 / 2,3 / 2,5 / 2$ are some less distinct wedges with period $T_{\text {sol }}=2 T_{f}$ (green). This structure resembles the Arnold tongues found in the Mathieu's differential equation, see e.g. [1]. The average time between pulses is not shown, but generally $\delta / T_{f}$ increases when moving down and to the right in figure 9 . Close to the $\theta$-axis, the tongue from $\theta=1$ have exactly $\delta=T_{f}$, whereas the tongues from $\theta=2,3$ have varying values around $\delta \approx 0.7 T_{f}, 0.45 T_{f}$, respectively.

\section{Discussion}

We have studied the effect of environmental variations on a physiologically structured population. This was done by taking a size structured model by Kooijman and Metz [4, 11] and introducing forcing, both when the unforced system exhibit an equilibrium solution (case A) and when it exhibits an oscillatory solution (case B). In each case, the amplitude and period of the forcing were varied systematically.

Environmental variations can be more or less regular, in both frequency, amplitude and shape. If the cycles of the variations are close to being periodic, 


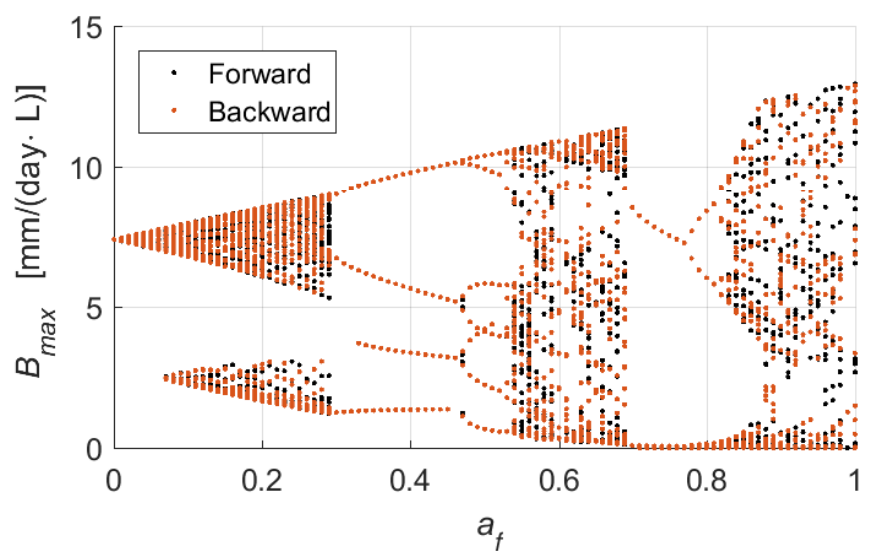

(a)

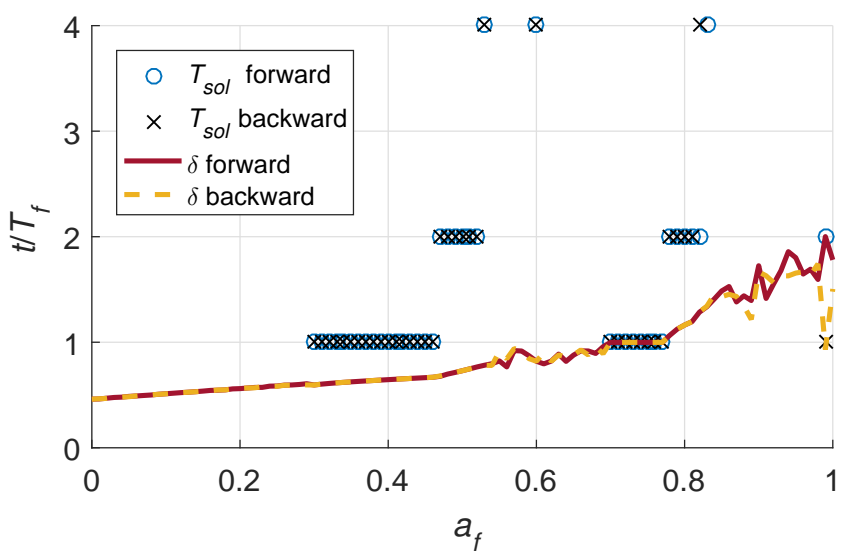

(b)

Figure 8: Parameter sweep in the forcing amplitude $a_{f}$ for case $\mathrm{B}(\mu=$ 0.25 day $^{-1}, K=0.7 \mathrm{mgC} / \mathrm{L}$ ) with a time scale ratio $\theta \approx 2.2$. (a) The local maxima $B_{\max }$ of the birth rate for each value of $a_{f}$. There are regions with periodic solutions and period doublings, and regions with irregular behavior. (b) The period $T_{\text {sol }}$ of the solution and the average time $\delta$ between birth pulses. There are two intervals with period $T_{\text {sol }}=T_{f}$, but with different values of $\delta$, implying a difference in the solutions. 


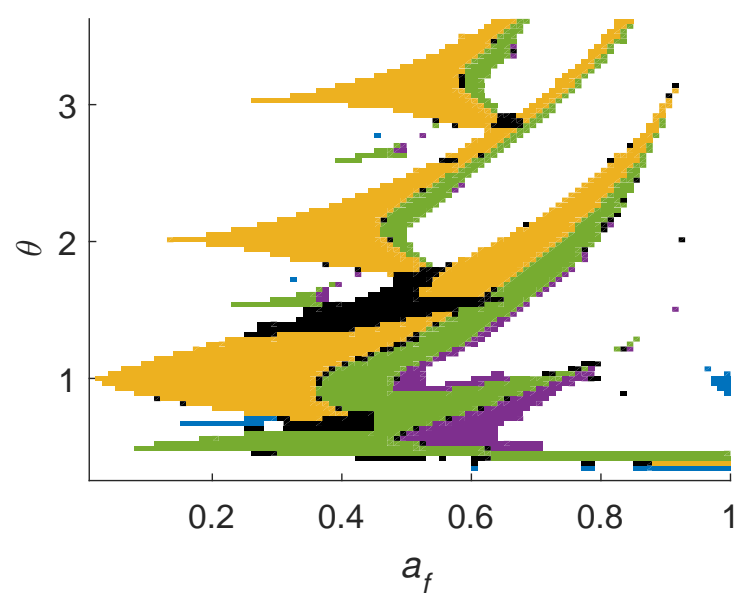

Figure 9: Bifurcation diagram for case $\mathrm{B}\left(\mu=0.25\right.$ day $\left.^{-1}, K=0.7 \mathrm{mgC} / \mathrm{L}\right)$ with parameters for the forcing amplitude $a_{f}$ and the time ratio $\theta=T_{f} / T_{\text {sys }}$. The colors indicate the solution period $T_{s o l}$ in units of $T_{f}, T_{s o l}=T_{f}$ is orange, $T_{\text {sol }}=2 T_{f}$ is green, $T_{\text {sol }}=3 T_{f}$ is blue, and $T_{\text {sol }}=4 T_{f}$ is purple. White indicates $T_{\text {sol }}>4 T_{f}$ or that no period was found, while black indicates the forward solution was different from the backward solution.

we expect the results presented here will still be applicable. In reality, some variations might be far from periodic and could even resemble random noise, and then a different approach would be needed. Here, we have chosen to focus on periodic variations.

In case $\mathrm{A}$, the unforced system has a stable equilibrium solution with oscillatory transient behavior, and the results of adding forcing are summarized in figure 5. When forcing with low amplitudes ( $a_{f}$ close to 0 ) is introduced, the population oscillates in phase with the forcing. When the forcing amplitude is increased, the system undergoes (one or more) period doublings, and thus show solutions with longer periods than that of the forcing. The period doublings occur for lower forcing amplitude when $\theta$ is close to 1, i.e., when the forcing frequency is close to the systems internal frequency. These results suggest that the population oscillates in phase with the environmental variations, though period doublings may occur, particularly near resonance frequencies.

The system in case B exhibits a stable periodic solution in the absence of forcing. The effects of forcing are summarized in figure 9 and resembles the classic pattern of Arnold tongues [1]. The tongues start at rational values of $\theta$ and represents windows of phase-locking. Period doublings occur as the forcing amplitude $a_{f}$ is increased. The broadest tongues are the ones starting at integer values of $\theta$, corresponding to forcing periods of $T_{f} \approx 27.6,55.2,82.7$ days. These tongues represent solutions that have the same period as the forcing (before period doublings), but with different number of birth pulses per cycle. Outside the tongues are regions with irregular (chaotic or quasi-periodic) solutions. These regions generally become more dominant when moving away from $\theta=1$.

Our findings agree with similar studies of forcing in predator-prey models without size structure. Forcing of the unstructured Rosenzweig-MacArthur 
model reveals bifurcations diagrams with Arnold tongues similar to figure 9, for parameters giving oscillatory solutions in the absence forcing, as in case B $[20,22]$. For parameters corresponding to case A, i.e., parameters where the unforced system displays oscillatory decay towards a stable equilibrium, forcing of the Rosenzweig-MacArthur model gives results resembling those in figure 5 $[20]$.

The tongues of phase-locking in case B start at rational values of the ratio $\theta=$ $T_{f} / T_{\text {sys }}$. We believe this pattern is general for environmental variations with period $T_{f}$ and populations with self-oscillations with period $T_{\text {sys }}$. Therefore, our results could also apply to populations of other species. For example, for a population with internal oscillation period of $T_{\text {sys }}=6$ months subjected to an annual variation, we have $\theta=2$ and thus expect the population to have two birth pulses repeating each year for a wide range of forcing amplitudes.

\section{Acknowledgements}

This work was supported by the Centre for Ocean Life, a VKR Centre of Excellence funded by the Villum Foundation.

\section{References}

[1] Vladimir I Arnold. Ordinary Differential Equations. Springer, 1992.

[2] COCO. Continuation Core and Toolboxes. https://sourceforge.net/ projects/cocotools.

[3] Harry Dankowicz and Frank Schilder. Recipes for continuation, volume 11. SIAM, 2013.

[4] AM De Roos, JAJ Metz, E Evers, and A Leipoldt. A size dependent predator-prey interaction: who pursues whom? Journal of Mathematical Biology, 28(6):609-643, 1990.

[5] André M de Roos. A gentle introduction to physiologically structured population models. In Structured-population models in marine, terrestrial, and freshwater systems, pages 119-204. Springer, 1997.

[6] André M de Roos and Lennart Persson. Population and community ecology of ontogenetic development. Princeton University Press, 2013.

[7] Geoffrey T Evans and John S Parslow. A model of annual plankton cycles. Biological oceanography, 3(3):327-347, 1985.

[8] Peter JS Franks. NPZ models of plankton dynamics: their construction, coupling to physics, and application. Journal of Oceanography, 58(2):379$387,2002$.

[9] Jan A Freund, Sebastian Mieruch, Bettina Scholze, Karen Wiltshire, and Ulrike Feudel. Bloom dynamics in a seasonally forced phytoplanktonzooplankton model: trigger mechanisms and timing effects. Ecological complexity, 3(2):129-139, 2006. 
[10] Arieh Iserles. A first course in the numerical analysis of differential equations. Cambridge University Press, 2009.

[11] SALM Kooijman and JAJ Metz. On the dynamics of chemically stressed populations: the deduction of population consequences from effects on individuals. Ecotoxicology and environmental safety, 8(3):254-274, 1984.

[12] Randall J LeVeque. Finite difference methods for ordinary and partial differential equations: steady-state and time-dependent problems, volume 98. Siam, 2007.

[13] Shang-keng Ma. Modern theory of critical phenomena. BenjaminCummings, 1976.

[14] MATLAB. Version 8.4.0.150421 (R2014b). The MathWorks Inc., Natick, Massachusetts, 2014.

[15] Bailey C McMeans, Kevin S McCann, Murray Humphries, Neil Rooney, and Aaron T Fisk. Food web structure in temporally-forced ecosystems. Trends in ecology \& evolution, 30(11):662-672, 2015.

[16] Johan A Metz and Odo Diekmann. The dynamics of physiologically structured populations, volume 68. Springer, 1986.

[17] Sergio Rinaldi, Simona Muratori, and Yuri Kuznetsov. Multiple attractors, catastrophes and chaos in seasonally perturbed predator-prey communities. Bulletin of mathematical Biology, 55(1):15-35, 1993.

[18] Michael L Rosenzweig et al. Paradox of enrichment: destabilization of exploitation ecosystems in ecological time. Science, 171(3969):385-387, 1971.

[19] Zepeng Sun and André M de Roos. Alternative stable states in a stagestructured consumer-resource biomass model with niche shift and seasonal reproduction. Theoretical population biology, 2015.

[20] Rachel A Taylor, Jonathan A Sherratt, and Andrew White. Seasonal forcing and multi-year cycles in interacting populations: lessons from a predator-prey model. Journal of mathematical biology, 67(6-7):1741-1764, 2013.

[21] HR Thieme. Well-posedness of physiologically structured population models for daphnia magna. Journal of Mathematical Biology, 26(3):299-317, 1988.

[22] John Vandermeer, Lewi Stone, and Bernd Blasius. Categories of chaos and fractal basin boundaries in forced predator-prey models. Chaos, Solitons $\&$ Fractals, 12(2):265-276, 2001.

[23] K. E. van de Wolfshaar, A. M. de Roos, and L. Persson. Population feedback after successful invasion leads to ecological suicide in seasonal environments. Ecology, 89(1):pp. 259-268, 2008. 